\title{
CONTRIBUTORS TO THIS ISSUE
}

Abdel-Malek H.A.

Albar H.M.

Asiri A.M.

Babu K.S.

Baumstark A.L.

Bhardwaj V.

Boyle P.H.

Dalloul H.M.

de Aguiar A.P.

Faidallah H.M.

Fozooni S.

Franklin P.J.

Gucky $\mathrm{T}$.

Harakishore $\mathrm{K}$.

Ito $\mathrm{K}$

Khalilullah M.D.

Kumar R.

Latha D.

Leggett-Robinson P.M.

Mahanti M.K.

Martinms P R C.

Molina C.T.

Morohashi N.

Murthy U.S.N.

Myrboh B.

Nagarapu L.

Navarro-Eisenstein A.M.
457

483

483

519

433,449

515

507

507

535

483

473

449

437

519

443

453

515

453

433

489

493

535

443

519

465

479

449
Nongkhlaw R.L.

465

Nongrum R.

465

Ohba Y.

443

Pallavi K.

Prakash O.

Praveen B.

Raju B.C.

Rao J.M.

Rao K.S.

Reddy G.J.

Reddy $P$.

Reddy P.S.N.

Romeiro G. A.

Sharma P.K.

Sharshira E.M.

Singh K.N.

Singh S.

Slouka J.

Srinivasulu B.

Suante $\mathrm{H}$.

Teraura $\mathrm{H}$.

Tikdari A.M.

Vasquez P.

Vásquez P.C.

Verma M.

Wiedermannová I.
453

515

519

519

519

503

453,503

503

503

493

515

483,519

499

499

437

479

489

443

473

433

449

499

437 